\title{
Theory of a resonantly interacting impurity in a Bose-Einstein condensate
}

\author{
Moritz Drescher, Manfred Salmhofer, and Tilman Enss () \\ Institut für Theoretische Physik, Universität Heidelberg, D-69120 Heidelberg, Germany
}

(Received 4 March 2020; accepted 22 June 2020; published 10 July 2020)

\begin{abstract}
We investigate a Bose-Einstein condensate in strong interaction with a single impurity particle. While this situation has received considerable interest in recent years, the regime of strong coupling remained inaccessible to most approaches due to an instability in the Bogoliubov theory arising near the resonance. We present a nonlocal extension of the Gross-Pitaevskii theory that is free of such divergences and does not require the use of the Born approximation in any of the interaction potentials. We find a new dynamical transition regime between attractive and repulsive polarons, where an interaction quench results in a finite number of coherent oscillations in the density profiles of the medium and in the contact parameter before equilibrium is reached.
\end{abstract}

DOI: 10.1103/PhysRevResearch.2.032011

Introduction. The motion of an impurity through a dynamical medium consisting of many interacting particles or field excitations is a fundamental physical problem. The impurity gets dressed by a cloud of excitations of the medium, which may enhance its mass (or even generate it in the first place, as in the Higgs effect) and also renormalize other properties $[1,2]$. Conversely, the medium is deformed in the vicinity of the impurity. This blurs the distinction between impurity and medium, and new many-body excitations and entangled states emerge [3]. In particular, an impurity subject to a strong attractive interaction with the particles in the medium may form bound states with them. Such many-body bound states allow one to probe the properties of the medium itself and occur, e.g., in ultracold gases [4,5], Rydberg systems [6], semiconductor heterostructures [7], or as dissipative bound states in photonic crystals and matter waves [8,9].

Recent experiments with ultracold quantum gases have probed impurity atoms in a Bose-Einstein condensate (BEC) medium, so-called Bose polarons [6,10-15]. In these systems, the impurity-boson (IB) interaction can be tuned by a Feshbach scattering resonance to strong attraction and even to the formation of two-body bound states between impurity and bosons [16]. While such bound states are well understood on the two-body level, they lead to a new competition on the many-body level: the short-range attraction tends to bind as many medium particles as possible to the impurity and strongly enhances the local medium density, while the short-range boson-boson (BB) repulsion between the medium particles counteracts this effect and tends to spread the bosons evenly. The competition between impurity-boson and bosonboson interaction can cause new dynamical many-body effects such as quantum flutter [17].

Published by the American Physical Society under the terms of the Creative Commons Attribution 4.0 International license. Further distribution of this work must maintain attribution to the author(s) and the published article's title, journal citation, and DOI.
Previous theoretical approaches based on the Fröhlich Hamiltonian [1,3,18-28] accurately describe the threedimensional (3D) Bose polaron at weak coupling. In order to capture the bound state at strong coupling, it is necessary to go beyond the Fröhlich model and include quadratic coupling terms [4,5,29-39]. Even then, the use of the Bogoliubov approximation misses cubic and quartic interaction terms, which are a crucial part of the boson repulsion required to balance the local attraction and prevent an unlimited growth of local density, as pointed out in [5,38]. Alternatively, GrossPitaevskii theory fully incorporates boson repulsion but does not describe bound states with a local interaction in Born approximation [40-42].

In this Rapid Communication, we develop a nonlocal extension of the Gross-Pitaevskii theory (GPT) that captures both competing effects on equal footing. This requires incorporating the explicit spatial form of the competing short-range attractive and repulsive interaction potentials. We apply our approach to study quench dynamics resulting from suddenly switching on the interaction between impurity and bosons: for this case, we predict stable and long-lived coherent oscillations of the medium density and correlations in space and time on the repulsive side of a scattering resonance; these are within reach of experimental observation. Near the (phase) boundary between the attractive and repulsive Bose polaron dynamics close to resonance, we find a new dynamical transition regime where a finite number of coherent oscillations occur before an attractive polaron is formed.

Model. The Hamiltonian of a Bose gas with a single impurity is, in first quantized form,

$$
\begin{aligned}
H= & \frac{\hat{\boldsymbol{p}}_{\mathrm{I}}^{2}}{2 m_{\mathrm{I}}}+\sum_{i} \frac{\hat{\boldsymbol{p}}_{\mathrm{B}, i}^{2}}{2 m_{\mathrm{B}}}+\sum_{i} V^{\mathrm{IB}}\left(\hat{\boldsymbol{x}}_{\mathrm{B}, i}-\hat{\boldsymbol{x}}_{\mathrm{I}}\right) \\
& +\sum_{i<j} V^{\mathrm{BB}}\left(\hat{\boldsymbol{x}}_{\mathrm{B}, i}-\hat{\boldsymbol{x}}_{\mathrm{B}, j}\right),
\end{aligned}
$$

where indices $I$ and $B$ stand for impurity and bosons with masses $m_{\mathrm{I}}$ and $m_{\mathrm{B}}$, respectively, and $i, j$ enumerate the bosons. 
We assume a repulsive BB interaction $V^{\mathrm{BB}}(\boldsymbol{x})>0$ and an attractive IB interaction $V^{\mathrm{IB}}(\boldsymbol{x})<0$.

For a single impurity it is convenient to use relative coordinates comoving with the impurity, making use of total momentum conservation. This is achieved by the Lee-Low-Pines (LLP) transformation [1,43] generated by $S=\hat{\boldsymbol{x}}_{I} \cdot \sum_{i} \hat{\boldsymbol{p}}_{B, i}$. Writing $\boldsymbol{p}_{0}$ for the conserved total momentum and $\boldsymbol{x}_{i}$ for the relative coordinate of the $i$ th boson, we obtain the LLP Hamiltonian $H_{\mathrm{LLP}}=\exp (i S) H \exp (-i S)$ :

$$
\begin{aligned}
H_{\mathrm{LLP}}= & \frac{\left(\boldsymbol{p}_{0}-\sum_{i} \hat{\boldsymbol{p}}_{i}\right)^{2}}{2 m_{\mathrm{I}}}+\sum_{i} \frac{\hat{\boldsymbol{p}}_{i}^{2}}{2 m_{\mathrm{B}}}+\sum_{i} V^{\mathrm{IB}}\left(\hat{\boldsymbol{x}}_{i}\right) \\
& +\sum_{i<j} V^{\mathrm{BB}}\left(\hat{\boldsymbol{x}}_{i}-\hat{\boldsymbol{x}}_{j}\right) .
\end{aligned}
$$

Gross-Pitaevskii theory in relative coordinates. The case of a long-range impurity potential $V^{\mathrm{IB}}$ can be described by standard GPT [44]. In particular, this includes interesting cases where the impurity potential has a bound state, which mimics the repulsive side of a Feshbach resonance. When this potential is suddenly switched on, long-lived coherent oscillations appear from the interference between the bound state and the homogeneous BEC state [38]. In the following, we first introduce GPT and discuss under which conditions it applies to the Hamiltonian (1); we then develop the new theory that works also for competing short-range potentials.

A heuristic derivation of GPT consists in replacing a radial $\mathrm{BB}$ potential by a local term in Born approximation, $V^{\mathrm{BB}}(r) \rightarrow\left(4 \pi a_{\mathrm{BB}} / m_{\mathrm{B}}\right) \delta^{(3)}(r)$, where $a_{\mathrm{BB}}$ is the scattering length, and making a product Ansatz

$$
\Psi\left(x_{1}, \ldots, x_{N}\right)=\phi\left(x_{1}\right) \cdots \phi\left(x_{N}\right),
$$

where $\phi$ is normalized as $\int d^{d} x|\phi(\boldsymbol{x})|^{2}=N$.

In the thermodynamic limit one derives the energy functional

$$
\begin{aligned}
E_{\mathrm{GP}}[\phi]= & \int_{\boldsymbol{x}}\left(\frac{|\nabla \phi|^{2}}{2 m_{\mathrm{red}}}+V^{\mathrm{IB}}|\phi|^{2}+\frac{4 \pi a_{\mathrm{BB}}}{2 m_{\mathrm{B}}}|\phi|^{4}\right) \\
& +\frac{\left(\boldsymbol{p}_{0}-\int \bar{\phi} \hat{\boldsymbol{p}} \phi\right)^{2}}{2 m_{\mathrm{I}}}
\end{aligned}
$$

with reduced mass $m_{\mathrm{red}}^{-1}=m_{\mathrm{I}}^{-1}+m_{\mathrm{B}}^{-1}$. It differs from the usual GP energy functional in three ways: (i) the external potential is replaced by the impurity potential, which is static in the LLP frame; (ii) the kinetic term contains the reduced mass instead of the boson mass, and (iii) the term in the second line induces correlations between bosons due to the motion of the impurity. It is nonconstant only when $\phi$ is not spherically symmetric: within the product Ansatz (2), this is the case only when the impurity is initially moving with respect to the condensate $\left(\boldsymbol{p}_{0} \neq 0\right)$.

Applicability of GPT to Bose polarons. GPT is usually applied to a Bose gas confined in an external potential that varies on a length scale larger than the range of the BB potential. This separation of scales justifies the Born approximation above. For the system at hand, this means that the energy functional (3) can only be applied when range of the impurity potential $V^{\mathrm{IB}}$ is large, but not for arbitrary potential shapes where the length scales no longer separate. In particular, a contact impurity interaction would require the boson field $\phi(x)$ to diverge as $1 /|\boldsymbol{x}|$ near the impurity $|\boldsymbol{x}| \rightarrow 0$, but then the BB term $\int_{x}|\phi(x)|^{4} \sim 1 / r_{0}$ diverges as the inverse of the potential range $r_{0}$. Hence, the standard GPT fails to describe competing short-range interactions as they occur in ultracold quantum gases.

If both potentials are weak, the Born approximation may be applied to $V^{\mathrm{IB}}$ as well, hence it works when the two interaction length scales are of the same order $[41,42]$. Here, however, we are interested in large IB coupling and need to look for a theory that takes into account the interaction potential shapes in more detail.

Generalization to strong coupling. GPT was rigorously proven to be exact for the ground state of a dilute gas, for a large class of repulsive BB potentials and without requiring the Born approximation [45]. An essential insight is that the wave function is not described by a pure product state (which would immediately lead to infinite energy in the case of a hard-sphere potential), but rather by $[45,46]$

$$
\Psi\left(x_{1}, \ldots, x_{N}\right)=\phi\left(x_{1}\right) \cdots \phi\left(x_{N}\right) F\left(x_{1}, \ldots, x_{N}\right) .
$$

Here, $F$ encodes two-body effects: $F=1$ when all particles are far apart from each other, but it behaves as the zero-energy scattering solution when any two particles are close to each other, and as a product of such two-body wave functions when multiple two-clusters occur. For a soft potential, $F \approx 1$ everywhere and the product state is recovered. Specifically, we choose

$$
F\left(\boldsymbol{x}_{1}, \ldots, \boldsymbol{x}_{N}\right)=\prod_{i<j} f\left(\boldsymbol{x}_{i}-\boldsymbol{x}_{j}\right),
$$

where $f$ is the zero-energy scattering solution of the twoboson problem, normalized as $\lim _{|x| \rightarrow \infty} f(x)=1$,

$$
\left(-\frac{\nabla^{2}}{m_{\mathrm{B}}}+V^{\mathrm{BB}}(\boldsymbol{x})\right) f(\boldsymbol{x})=0 .
$$

For instance, a hard-sphere potential of range $a_{\mathrm{BB}}$ has $f(\boldsymbol{x})=$ $\max \left(0,1-a_{\mathrm{BB}} /|\boldsymbol{x}|\right)$. The two-body correlated wave functions (4) were first employed by Jastrow [47], and Ansätze of similar type are used in rigorous studies of BEC dynamics [48-50] and in quantum Monte Carlo calculations. Taking the expectation value of the Hamiltonian (1) in a correlated wave function of the form (4), we obtain the energy functional $E[\phi]=\langle\Psi|H| \Psi\rangle /\langle\Psi \mid \Psi\rangle$ in the low-density limit as the first main result of this work,

$$
\begin{aligned}
E[\phi]= & \frac{\left(\boldsymbol{p}_{0}-\int \bar{\phi} \hat{\boldsymbol{p}} \phi\right)^{2}}{2 m_{\mathrm{I}}}+\int_{\boldsymbol{x}}\left(\frac{|\nabla \phi|^{2}}{2 m_{\mathrm{red}}}+V^{\mathrm{IB}}|\phi|^{2}\right) \\
& +\int_{\boldsymbol{x}_{1}, \boldsymbol{x}_{2}}\left|\phi_{1} \phi_{2}\right|^{2}\left[\frac{f_{12}^{\prime 2}}{2 m_{\mathrm{B}}}+\frac{V_{12}^{\mathrm{BB}}}{2} f_{12}^{2}\right] \\
& +\operatorname{Re} \int_{\boldsymbol{x}_{1}, \boldsymbol{x}_{2}} \frac{\overline{\phi_{1}} \boldsymbol{\nabla} \phi_{1} \cdot \overline{\phi_{2}} \nabla \phi_{2}\left(1-f_{12}^{2}\right)}{2 m_{\mathrm{I}}}
\end{aligned}
$$

with $\phi_{1}=\phi\left(\boldsymbol{x}_{1}\right), f_{12}=f\left(\boldsymbol{x}_{1}-\boldsymbol{x}_{2}\right)$, etc., and $f^{\prime}$ is the derivative of $f$ (since $V^{\mathrm{BB}}$ is radially symmetric, $f$ only depends on $|\boldsymbol{x}|)$. The most important difference to the original GP functional (3) is the BB term (second line), which is now a double integral and involves not only the scattering length but the full BB potential. The two integrals decouple only 
for weak IB coupling where $\phi_{1} \approx \phi_{2}$ and the square brackets yield the Born approximation $2 \pi a_{\mathrm{BB}} / m_{\mathrm{B}}$ [45]. At strong coupling, instead, the pair correlations contained in $f(x)$ are crucial to render the short-range interaction finite. The last line captures the back-reaction of the medium onto the impurity, which arises from correlations beyond the product state and is nonzero also for an impurity initially at rest $\left(\boldsymbol{p}_{0}=0\right)$. Few-body correlations are predicted to have a strong effect on attractive Bose polarons and yield universal properties when both $V^{\mathrm{IB}}$ and $V^{\mathrm{BB}}$ are short ranged [51,52].

Dynamics. We apply (5) to the situation where an impurity is suddenly quenched from a noninteracting to an interacting state, as can be realized by a hyperfine transition in experiment. We assume that the wave function retains the correlated form (4) with $f(\boldsymbol{x})$ fixed in time because the boson-boson pair correlation is always present in the condensate, also far away from the impurity. In (4), the time dependence of the impurityboson correlation is captured by $\phi(\boldsymbol{x}, t)$. This is analogous to the derivation of the dynamical GP equations from the GP energy functional; the corresponding nonlinear Schrödinger equation is obtained by varying $\mathcal{L}=\left\langle\Psi\left|i \partial_{t}-H\right| \Psi\right\rangle /\langle\Psi \mid \Psi\rangle$ with respect to $\phi$. This yields

$$
\begin{aligned}
i \partial_{t} \phi_{1}= & -\frac{\nabla^{2} \phi_{1}}{2 m_{\mathrm{red}}}+V_{1}^{\mathrm{IB}} \phi_{1}+\phi_{1} \int_{\boldsymbol{x}_{2}}\left|\phi_{2}\right|^{2}\left[\frac{f_{12}^{\prime 2}}{m_{\mathrm{B}}}+V_{12}^{\mathrm{BB}} f_{12}^{2}\right] \\
& +\frac{i \nabla \phi_{1}}{m_{\mathrm{I}}} \cdot\left(\boldsymbol{p}_{0}-\operatorname{Im} \int_{\boldsymbol{x}_{2}} \overline{\phi_{2}} \nabla \phi_{2} f_{12}^{2}\right) \\
& +\frac{\phi_{1}}{m_{\mathrm{I}}} \int_{\boldsymbol{x}_{2}} \phi_{2} \nabla \overline{\phi_{2}} \cdot f_{12} f_{12}^{\prime} \frac{\boldsymbol{x}_{2}-\boldsymbol{x}_{1}}{\left|\boldsymbol{x}_{2}-\boldsymbol{x}_{1}\right|} .
\end{aligned}
$$

We solve this equation numerically with the initial condition of a flat BEC, $\phi(x, t=0)=\sqrt{n_{0}}$, and an impurity at rest, $\boldsymbol{p}_{0}=0$. For the interaction potentials, we take $V^{\mathrm{BB}}$ to be either a Gaussian potential of depth $V_{0}$ and range $\sigma_{\mathrm{BB}}$,

$$
V^{\mathrm{BB}}(r)=V_{0} \exp \left(-r^{2} / \sigma_{\mathrm{BB}}^{2}\right),
$$

or a hard-sphere potential, while $V^{\mathrm{IB}}$ is modeled as a contact interaction using the 3D Fermi pseudopotential with scattering length $a_{\mathrm{IB}}$,

$$
V^{\mathrm{IB}}(r) \phi(r)=\frac{2 \pi a_{\mathrm{IB}}}{m_{\mathrm{red}}} \delta^{(3)}(r) \frac{\partial}{\partial r} r \phi(r) .
$$

This corresponds to the Bethe-Peierls boundary condition for $t>0$,

$$
\lim _{r \rightarrow 0} r \phi(r)+a_{\mathrm{IB}} \partial_{r} r \phi(r)=0 .
$$

The 3D contact potential is always attractive and has no bound state for weak attraction where $a_{\mathrm{IB}}<0$ (attractive side of Feshbach resonance). For strong attraction beyond the scattering resonance $1 / a_{\mathrm{IB}}=0$, there is a single bound state of energy $E_{B}=\left(2 m_{\mathrm{red}} a_{\mathrm{IB}}^{2}\right)^{-1}$ and the scattering length $a_{\mathrm{IB}}>0$ is positive (repulsive side) [16].

Results. After an interaction quench, the boson density profile exhibits two distinct phenomenologies: (i) a density profile enhanced with respect to $n_{0}$ that converges for long times ("attractive polaron"), and (ii) a profile depleted in a "halo" around the impurity that oscillates in time ("repulsive polaron"). Previous approaches based on the Bogoliubov theory $[34,38]$ have shown that quenches to the attractive side
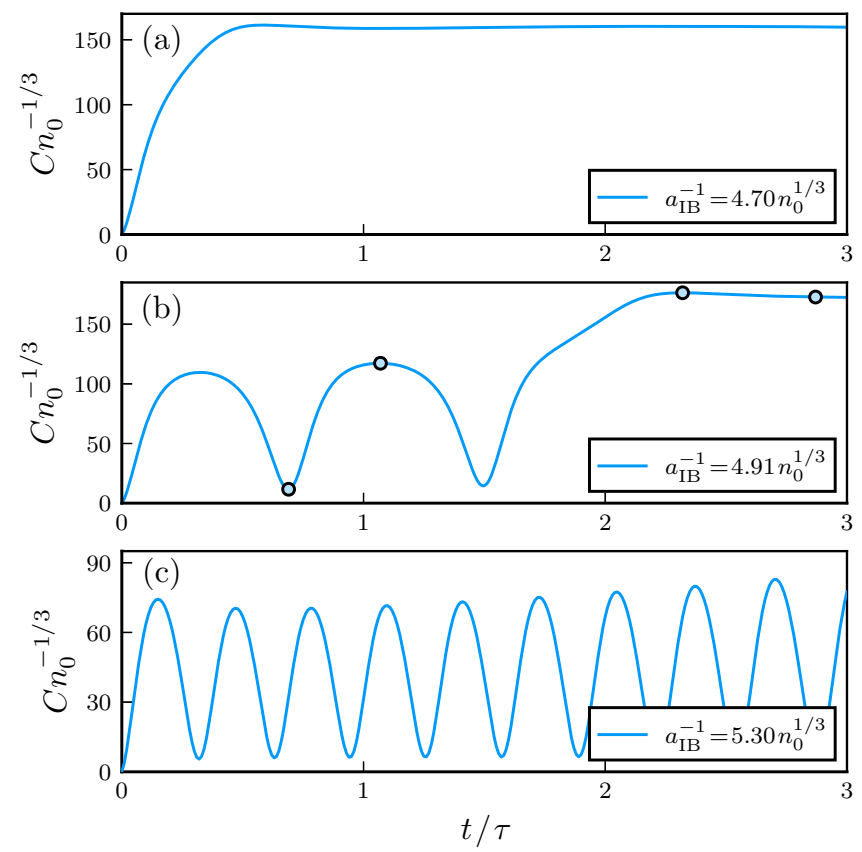

FIG. 1. The contact parameter $C$ as a function of time for three different IB interaction strengths. (a) No oscillations are observed despite the positive scattering length; the polaron is effectively attractive due to the medium shift of the resonance in the presence of BB interactions. (b) Close to the transition point, the system performs a limited number of oscillations before converging, featuring a dynamical transition from a repulsive to an attractive polaron. (c) Further on the repulsive side, the system keeps oscillating in agreement with previous predictions [38]. Calculations were carried out at $a_{\mathrm{BB}}=$ $0.03 n_{0}^{-1 / 3}, \sigma_{\mathrm{BB}}=0.1 n_{0}^{-1 / 3}$, and $m_{\mathrm{B}}=m_{\mathrm{I}}$. Time is measured in units of the BEC timescale $\tau=m_{\mathrm{B}} n_{0}^{-2 / 3} / \hbar$.

of the Feshbach resonance yield attractive polarons, while repulsive polarons form further on the repulsive side of the resonance; however, near the Feshbach resonance a dynamical instability arises. The present approach is free of such instabilities, which allows us to investigate the transition region near the resonance. We find that the transition between the many-body attractive and repulsive polaron states is shifted toward the repulsive side of the scattering resonance due to the many-body environment $[34,38]$. Our results for equal mass $m_{\mathrm{B}}=m_{\mathrm{I}}$ are representative of the mass imbalanced case within the general theory (5).

Transition from convergent to oscillatory dynamics. In Fig. 1, we show the time evolution of Tan's contact parameter $C=\left|4 \pi \lim _{r \rightarrow 0} r \phi(r)\right|^{2}[15,53]$, which quantifies the probability to find bosons near the impurity and determines the short-distance singularity of the pair-correlation function $g_{\mathrm{IB}}^{(2)}(\boldsymbol{r})=\int_{\boldsymbol{R}}\left\langle\hat{n}_{\mathrm{B}}(\boldsymbol{R}+\boldsymbol{r}) \hat{n}_{\mathrm{I}}(\boldsymbol{R})\right\rangle=C /(4 \pi r)^{2}+O(1 / r)$, where $\hat{n}_{\mathrm{I}}(\boldsymbol{x})=\delta\left(\boldsymbol{x}-\hat{\boldsymbol{x}}_{\mathrm{I}}\right)$ and $\hat{n}_{\mathrm{B}}(\boldsymbol{x})=\sum_{i} \delta\left(\boldsymbol{x}-\hat{\boldsymbol{x}}_{\mathrm{B}, i}\right)$ denote the impurity and boson density operators, respectively. For an ideal BEC, the transition between convergent and oscillatory behavior is exactly at the resonance $a_{\mathrm{IB}}^{-1}=0$ and the frequency is given by the binding energy $E_{B}$ of one boson to the impurity [38]. In the presence of $\mathrm{BB}$ interactions, the boundary is shifted toward the repulsive side and convergence persists at large positive scattering lengths, as shown in Fig. 1(a). In the 

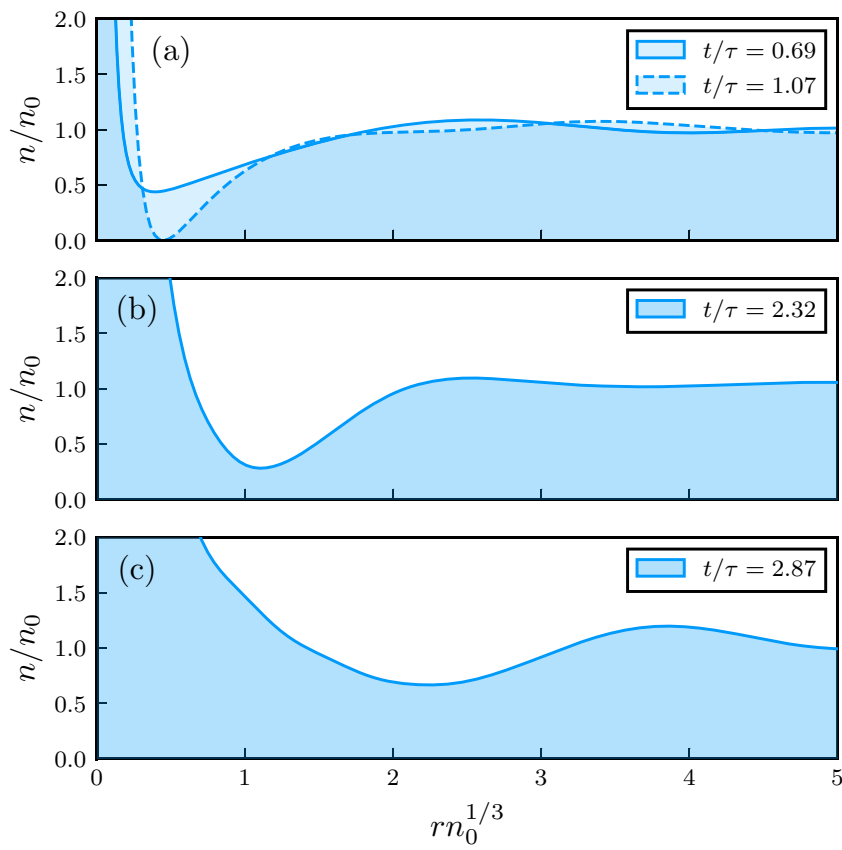

FIG. 2. BEC density profiles at different times in the transition regime. The points in time and parameters are those indicated in Fig. 1(b), while $r$ denotes the distance from the impurity. One can see the dynamical transition from a repulsive to an attractive polaron: At short times (a), a halo of depletion around the impurity forms and oscillates in depth [38]. At intermediate times, the depletion hole moves outwards and becomes more shallow (b). Finally, the profile of an attractive polaron is approached (c).

transition region, the system performs only a finite number of oscillations before assuming convergent behavior [Fig. 1(b)]. This critical region is narrow and the number of oscillations grows rapidly as the scattering length is tuned further to the repulsive side [Fig. 1(c)].

The dynamical transition in Fig. 1(b) is clearly visible also in the density profiles of the BEC around the impurity (Fig. 2): for short times the condensate density is strongly depleted in a halo around the impurity that oscillates in space and time, typical of a repulsive polaron. For later times, instead, the depletion propagates outwards and the density profiles of the BEC attain the shape of an attractive polaron with typically $\Delta N=10 \ldots 100$ extra bosons in the dressing cloud of the impurity [38]. This dynamical transition regime was inaccessible with previous approaches based on the Bogoliubov approximation and is the second main result of this work.

Position of the resonance in medium and dependence on potential shape. For competing short-range interactions, the question arises whether the $\mathrm{BB}$ interaction is characterized by its scattering length alone, as in the GP and Bogoliubov theories, or whether further details of the potential need to be taken into account. To answer this question, we compute the oscillation frequency after a quench close to the resonance for different BB potential shapes with the same scattering length but different height and width, as shown in Fig. 3 (inset). For weak IB coupling $a_{\mathrm{IB}} \ll n_{0}^{-1 / 3}$ the curves converge, demonstrating that the $\mathrm{BB}$ interaction is indeed fully characterized by

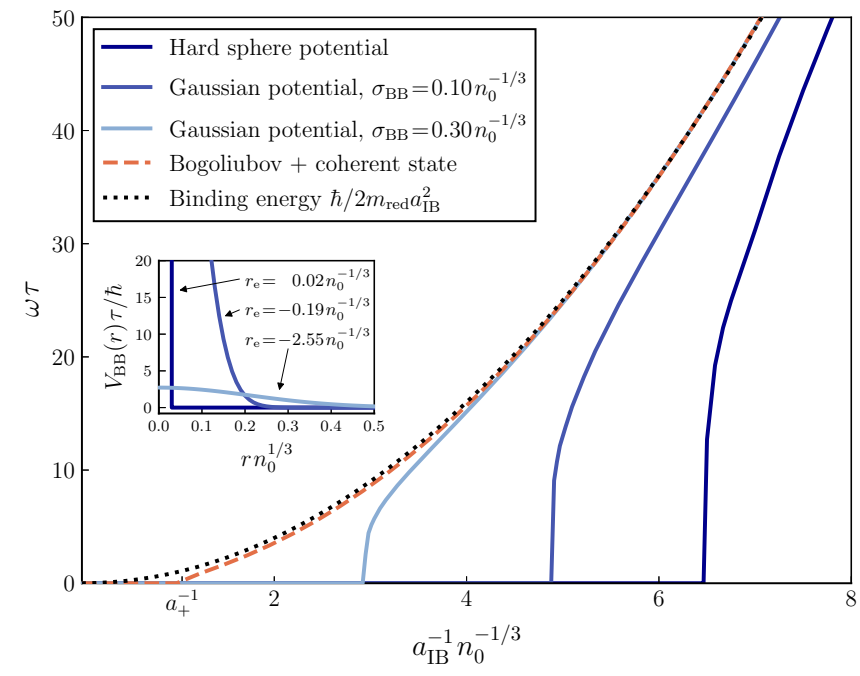

FIG. 3. Frequency of coherent oscillations vs impurity-boson coupling strength, for different BB potentials with the same scattering length $a_{\mathrm{BB}}=0.03 n_{0}^{-1 / 3}$ but different shapes and effective range $r_{\mathrm{e}}$ (see inset). The onset of the oscillations marks the transition from an attractive to a repulsive polaron. We find that the transition point depends on the shape of the $\mathrm{BB}$ potential and is shifted further toward the repulsive side for a hard-core interaction (black line); different IB ranges yield qualitatively similar results. Softer BB potentials (gray), instead, approach the Bogoliubov result (dashed) [38] with mean-field shifted resonance position $a_{+}^{-1}$ [34]. For weaker coupling, the oscillation frequencies approach the two-body binding energy $E_{B}$ (dotted) [38]. Parameters and units as in Fig. 1.

the scattering length alone. Closer to the resonance, however, the curves differ significantly. In particular, the transition point between attractive and repulsive polaron that marks the onset of oscillations is shifted much further into the repulsive regime for a hard-sphere potential than for one that is soft and long range. The result obtained from the Bogoliubov theory with a coherent-state ansatz [38] is recovered in the limit of soft potentials where the Born approximation is valid. In this case, the transition occurs at the mean-field shifted resonance position $a_{\mathrm{IB}}=a_{+}[5,34,38,54]$ as indicated in Fig. 3.

To conclude, we have developed a nonlocal extension (5) of Gross-Pitaevskii theory that is able to treat the impurityBEC problem at large coupling strengths $a_{\mathrm{IB}} \sim n_{0}^{-1 / 3}$ also close to a Feshbach resonance. We find a new dynamical transition region between attractive and repulsive polarons, where the BEC density profile performs a finite number of oscillations near the impurity before converging to an attractive polaron profile. These phenomena might be observed with current experiments in ultracold atomic gases $[6,12,13,15]$ in combination with ultrafast interferometry [55], which can resolve oscillation periods of $100 \ldots 1000 \mu \mathrm{s}$ estimated for lithium-cesium mixtures. The medium interaction shifts the interesting transition region further away from the resonance toward the repulsive side, and this makes it even easier to observe before three-body recombination occurs on typical timescales of several milliseconds [15]. Our approach captures the medium back-reaction, which could 
not be described by GPT or Bogoliubov theory with coherent variational states, and therefore applies also to ultracold atomic gases with mobile impurities. In particular, generalizations to a larger number of mobile impurities, where the impurity statistics start to matter, will describe also induced interactions between impurities mediated by the BEC [56]. More generally, we expect our methods to apply in systems with bound states and a linear excitation spectrum, where they provide a generic framework for impurity dressing in medium.
Note added. Recently, a complementary theoretical work appeared that employs the GPT for a finite-range IB potential to study static Bose polaron properties [57].

Acknowledgments. We thank N. Defenu, A. Imamoglu, R. Schmidt, and M. W. Zwierlein for stimulating discussions. This work is supported by Deutsche Forschungsgemeinschaft (DFG, German Research Foundation) via Collaborative Research Centre "SFB1225" (ISOQUANT) and under Germany's Excellence Strategy "EXC-2181/1-390900948" (the Heidelberg STRUCTURES Excellence Cluster).
[1] M. Girardeau, Motion of an impurity particle in a boson superfluid, Phys. Fluids 4, 279 (1961).

[2] J. T. Devreese and A. S. Alexandrov, Fröhlich polaron and bipolaron: Recent developments, Rep. Prog. Phys. 72, 066501 (2009).

[3] D. Boyanovsky, D. Jasnow, X.-L. Wu, and R. C. Coalson, Dynamics of relaxation and dressing of a quenched Bose polaron, Phys. Rev. A 100, 043617 (2019).

[4] S. P. Rath and R. Schmidt, Field-theoretical study of the Bose polaron, Phys. Rev. A 88, 053632 (2013).

[5] F. Grusdt, R. Schmidt, Y. E. Shchadilova, and E. Demler, Strong-coupling Bose polarons in a Bose-Einstein condensate, Phys. Rev. A 96, 013607 (2017).

[6] F. Camargo, R. Schmidt, J. D. Whalen, R. Ding, G. Woehl, S. Yoshida, J. Burgdörfer, F. B. Dunning, H. R. Sadeghpour, E. Demler, and T. C. Killian, Creation of Rydberg Polarons in a Bose Gas, Phys. Rev. Lett. 120, 083401 (2018).

[7] M. Sidler, P. Back, O. Cotlet, A. Srivastava, T. Fink, M. Kroner, E. Demler, and A. Imamoglu, Fermi polaron-polaritons in charge-tunable atomically thin semiconductors, Nat. Phys. 13, 255 (2017).

[8] Y. Liu and A. A. Houck, Quantum electrodynamics near a photonic bandgap, Nat. Phys. 13, 48 (2017).

[9] L. Krinner, M. Stewart, A. Pazmino, J. Kwon, and D. Schneble, Spontaneous emission of matter waves from a tunable open quantum system, Nature (London) 559, 589 (2018).

[10] J. Catani, G. Lamporesi, D. Naik, M. Gring, M. Inguscio, F. Minardi, A. Kantian, and T. Giamarchi, Quantum dynamics of impurities in a one-dimensional Bose gas, Phys. Rev. A 85, 023623 (2012).

[11] T. Rentrop, A. Trautmann, F. A. Olivares, F. Jendrzejewski, A. Komnik, and M. K. Oberthaler, Observation of the Phononic Lamb Shift with a Synthetic Vacuum, Phys. Rev. X 6, 041041 (2016).

[12] M.-G. Hu, M. J. Van de Graaff, D. Kedar, J. P. Corson, E. A. Cornell, and D. S. Jin, Bose Polarons in the Strongly Interacting Regime, Phys. Rev. Lett. 117, 055301 (2016).

[13] N. B. Jørgensen, L. Wacker, K. T. Skalmstang, M. M. Parish, J. Levinsen, R. S. Christensen, G. M. Bruun, and J. J. Arlt, Observation of Attractive and Repulsive Polarons in a BoseEinstein Condensate, Phys. Rev. Lett. 117, 055302 (2016).

[14] L. A. Peña Ardila, N. B. Jørgensen, T. Pohl, S. Giorgini, G. M. Bruun, and J. J. Arlt, Analyzing a Bose Polaron Across Resonant Interactions, Phys. Rev. A 99, 063607 (2019).

[15] Z. Z. Yan, Y. Ni, C. Robens, and M. W. Zwierlein, Bose polarons near quantum criticality, Science 368, 190 (2020).
[16] C. Chin, R. Grimm, P. Julienne, and E. Tiesinga, Feshbach resonances in ultracold gases, Rev. Mod. Phys. 82, 1225 (2010).

[17] C. J. M. Mathy, M. B. Zvonarev, and E. Demler, Quantum flutter of supersonic particles in one-dimensional quantum liquids, Nat. Phys. 8, 881 (2012).

[18] H. Fröhlich, Electrons in lattice fields, Adv. Phys. 3, 325 (1954).

[19] G. E. Astrakharchik and L. P. Pitaevskii, Motion of a heavy impurity through a Bose-Einstein condensate, Phys. Rev. A 70, 013608 (2004).

[20] F. M. Cucchietti and E. Timmermans, Strong-Coupling Polarons in Dilute Gas Bose-Einstein Condensates, Phys. Rev. Lett. 96, 210401 (2006).

[21] M. Bruderer, W. Bao, and D. Jaksch, Self-trapping of impurities in Bose-Einstein condensates: Strong attractive and repulsive coupling, Europhys. Lett. 82, 30004 (2008).

[22] J. Tempere, W. Casteels, M. K. Oberthaler, S. Knoop, E. Timmermans, and J. T. Devreese, Feynman path-integral treatment of the BEC-impurity polaron, Phys. Rev. B 80, 184504 (2009).

[23] W. Casteels, T. Van Cauteren, J. Tempere, and J. T. Devreese, Strong coupling treatment of the polaronic system consisting of an impurity in a condensate, Laser Phys. 21, 1480 (2011).

[24] A. Shashi, F. Grusdt, D. A. Abanin, and E. Demler, Radiofrequency spectroscopy of polarons in ultracold Bose gases, Phys. Rev. A 89, 053617 (2014).

[25] F. Grusdt, Y. E. Shchadilova, A. N. Rubtsov, and E. Demler, Renormalization group approach to the Fröhlich polaron model: Application to impurity-BEC problem, Sci. Rep. 5, 12124 (2015).

[26] J. Vlietinck, W. Casteels, K. van Houcke, J. Tempere, J. Ryckebusch, and J. T. Devreese, Diagrammatic Monte Carlo study of the acoustic and the Bose-Einstein condensate polaron, New J. Phys. 17, 033023 (2015).

[27] A. Lampo, S. H. Lim, M. Á. García-March, and M. Lewenstein, Bose polaron as an instance of quantum Brownian motion, Quantum 1, 30 (2017).

[28] K. K. Nielsen, L. A. P. Ardila, G. M. Bruun, and T. Pohl, Critical slowdown of non-equilibrium polaron dynamics, New J. Phys. 21, 043014 (2019).

[29] W. Li and S. Das Sarma, Variational study of polarons in Bose-Einstein condensates, Phys. Rev. A 90, 013618 (2014).

[30] R. S. Christensen, J. Levinsen, and G. M. Bruun, Quasiparticle Properties of a Mobile Impurity in a Bose-Einstein Condensate, Phys. Rev. Lett. 115, 160401 (2015). 
[31] J. Levinsen, M. M. Parish, and G. M. Bruun, Impurity in a Bose-Einstein Condensate and the Efimov Effect, Phys. Rev. Lett. 115, 125302 (2015).

[32] L. A. Peña Ardila and S. Giorgini, Impurity in a Bose-Einstein condensate: Study of the attractive and repulsive branch using quantum Monte Carlo methods, Phys. Rev. A 92, 033612 (2015).

[33] A. G. Volosniev, H.-W. Hammer, and N. T. Zinner, Real-time dynamics of an impurity in an ideal Bose gas in a trap, Phys. Rev. A 92, 023623 (2015).

[34] Y. E. Shchadilova, R. Schmidt, F. Grusdt, and E. Demler, Quantum Dynamics of Ultracold Bose Polarons, Phys. Rev. Lett. 117, 113002 (2016).

[35] F. Grusdt, K. Seetharam, Y. Shchadilova, and E. Demler, Strong-coupling Bose polarons out of equilibrium: Dynamical renormalization-group approach, Phys. Rev. A 97, 033612 (2018).

[36] B. Kain and H. Y. Ling, Analytical study of static beyondFröhlich Bose polarons in one dimension, Phys. Rev. A 98, 033610 (2018).

[37] T. Lausch, A. Widera, and M. Fleischhauer, Prethermalization in the cooling dynamics of an impurity in a Bose-Einstein condensate, Phys. Rev. A 97, 023621 (2018).

[38] M. Drescher, M. Salmhofer, and T. Enss, Real-space dynamics of attractive and repulsive polarons in Bose-Einstein condensates, Phys. Rev. A 99, 023601 (2019).

[39] S. I. Mistakidis, G. C. Katsimiga, G. M. Koutentakis, T. Busch, and P. Schmelcher, Quench Dynamics and Orthogonality Catastrophe of Bose Polarons, Phys. Rev. Lett. 122, 183001 (2019).

[40] P. Massignan, C. J. Pethick, and H. Smith, Static properties of positive ions in atomic Bose-Einstein condensates, Phys. Rev. A 71, 023606 (2005).

[41] A. A. Blinova, M. G. Boshier, and E. Timmermans, Two polaron flavors of the Bose-Einstein condensate impurity, Phys. Rev. A 88, 053610 (2013).

[42] J. Takahashi, R. Imai, E. Nakano, and K. Iida, Bose polaron in spherical trap potentials: Spatial structure and quantum depletion, Phys. Rev. A 100, 023624 (2019).

[43] T. D. Lee, F. E. Low, and D. Pines, The motion of slow electrons in a polar crystal, Phys. Rev. 90, 297 (1953).
[44] L. P. Pitaevskii and S. Stringari, Bose-Einstein Condensation (Oxford University Press, Oxford, 2003).

[45] E. H. Lieb, R. Seiringer, and J. Yngvason, Bosons in a trap: A rigorous derivation of the Gross-Pitaevskii energy functional, Phys. Rev. A 61, 043602 (2000).

[46] F. J. Dyson, Ground-state energy of a hard-sphere gas, Phys. Rev. 106, 20 (1957).

[47] R. Jastrow, Many-body problem with strong forces, Phys. Rev. 98, 1479 (1955).

[48] L. Erdős, B. Schlein, and H.-T. Yau, Derivation of the cubic non-linear Schrödinger equation from quantum dynamics of many-body systems, Invent. Math. 167, 515 (2007).

[49] L. Erdős, B. Schlein, and H.-T. Yau, Rigorous derivation of the Gross-Pitaevskii equation with a large interaction potential, J. Am. Math. Soc. 22, 1099 (2009).

[50] C. Brennecke and B. Schlein, Gross-Pitaevskii dynamics for Bose-Einstein condensates, Anal. PDE 12, 1513 (2019).

[51] S. M. Yoshida, S. Endo, J. Levinsen, and M. M. Parish, Universality of an Impurity in a Bose-Einstein Condensate, Phys. Rev. X 8, 011024 (2018).

[52] Z.-Y. Shi, S. M. Yoshida, M. M. Parish, and J. Levinsen, Impurity-Induced Multibody Resonances in a Bose Gas, Phys. Rev. Lett. 121, 243401 (2018).

[53] S. Tan, Energetics of a strongly correlated Fermi gas, Ann. Phys. (Amsterdam) 323, 2952 (2008).

[54] The mean-field shift of the resonance position is given by $a_{+}^{-1}=(2 / \pi) \int_{0}^{\infty} d k\left[1-\left(m_{\mathrm{I}}+m_{\mathrm{B}}\right) /\left(\gamma_{k}^{2} m_{\mathrm{I}}+\gamma_{k} m_{\mathrm{B}}\right)\right]$, where $\gamma_{k}=\sqrt{1+16 \pi a_{\mathrm{BB}} n_{0} / k^{2}}$.

[55] M. Cetina et al., Ultrafast many-body interferometry of impurities coupled to a Fermi sea, Science 354, 96 (2016).

[56] A. Camacho-Guardian and G. M. Bruun, Landau Effective Interaction between Quasiparticles in a Bose-Einstein Condensate, Phys. Rev. X 8, 031042 (2018).

[57] N.-E. Guenther, R. Schmidt, G. M. Bruun, V. Gurarie, and P. Massignan, Mobile impurity in a Bose-Einstein condensate and the orthogonality catastrophe, arXiv:2004.07166. 